University of Nebraska - Lincoln

DigitalCommons@University of Nebraska - Lincoln

Publications from USDA-ARS / UNL Faculty

U.S. Department of Agriculture: Agricultural

Research Service, Lincoln, Nebraska

2013

Soil quality response to long-term tillage and crop rotation practices

Douglas Karlen

USDA-ARS, doug.karlen@ars.usda.gov

Cynthia A. Cambardella

USDA-ARS, cindy.cambardella@ars.usda.gov

John L. Kovar

USDA-ARS, john.kovar@ars.usda.gov

Thomas S. Colvin

USDA-ARS, oxfrdfrm@huxcomm.net

Follow this and additional works at: https://digitalcommons.unl.edu/usdaarsfacpub

Karlen, Douglas; Cambardella, Cynthia A.; Kovar, John L.; and Colvin, Thomas S., "Soil quality response to long-term tillage and crop rotation practices" (2013). Publications from USDA-ARS / UNL Faculty. 1633. https://digitalcommons.unl.edu/usdaarsfacpub/1633

This Article is brought to you for free and open access by the U.S. Department of Agriculture: Agricultural Research Service, Lincoln, Nebraska at DigitalCommons@University of Nebraska - Lincoln. It has been accepted for inclusion in Publications from USDA-ARS / UNL Faculty by an authorized administrator of DigitalCommons@University of Nebraska - Lincoln. 


\title{
Soil quality response to long-term tillage and crop rotation practices
}

\author{
Douglas L. Karlen $^{\text {a,* }}$, Cynthia A. Cambardella ${ }^{\text {, }}$ John L. Kovar ${ }^{\text {a }}$, Thomas S. Colvin ${ }^{\mathrm{b}, 1}$ \\ a USDA-Agricultural Research Service (ARS), National Laboratory for Agriculture and the Environment (NLAE), 2110 University Boulevard, Ames, IA 50011- \\ 3120, United States \\ ${ }^{\mathrm{b}}$ USDA-ARS, 55670 290th Street, Cambridge, IA 50046, United States
}

\section{A R T I C L E I N F O}

\section{Article history:}

Received 11 March 2013

Received in revised form 29 May 2013

Accepted 30 May 2013

\section{Keywords:}

Conservation tillage

Basic cation saturation ratio (BCSR)

Maize

Soybean

Soil-testing

Soil properties

\begin{abstract}
A B S T R A C T
Soil quality is influenced by inherent and anthropogenic factors. This study was conducted to provide multiple groups guidance on how to achieve and maintain improved soil quality/health. Our hypothesis was that tillage intensity was the primary anthropogenic factor degrading soil quality, and our objective was to prove that hypothesis through an intensive 2005 sampling of a central Iowa, USA field study. Chisel plow, disk tillage, moldboard plow, ridge-till and no-till treatments, used for 31 years in a twoyear, corn (Zea mays L.)/soybean [Glycine max (L.) Merr.] (C/S) rotation or for 26 years of continuous corn (CC) production, were evaluated by measuring 23 potential soil quality indicators. Soil samples from 0 to 5 - and 5 to $15-\mathrm{cm}$ depth increments were collected from 158 loam or clay loam sampling sites throughout the 10-ha study site. Nine of the indicators were evaluated by depth increment using the Soil Management Assessment Framework (SMAF) which has scoring functions for 13 soil biological, chemical, and physical measurements and can be used to compute individual indicator indices and an overall soil quality index (SQI). Water-stable aggregation (WSA), total organic carbon (TOC), microbial biomass carbon (MBC), and potentially mineralizable nitrogen (PMN) were all significantly lower for the 0 to $5-\mathrm{cm}$ and generally lower for 5 to $15-\mathrm{cm}$ increments after long-term moldboard plowing and its associated secondary tillage operations. This presumably reflected greater physical breakup and oxidation of above- and below-ground plant residues. Bray-P concentrations in moldboard plow plots were also significantly lower at both depth increments. Between soil texture groups, significant differences were found for WSA, Bray-P, TOC and MBC at both depth increments and for both cropping systems. When combined into an overall SQI, both soil texture groups were functioning at $82-85 \%$ of their potential at $0-5-\mathrm{cm}$ and at $75 \%$ of their potential at the $5-15-\mathrm{cm}$ depth. Our hypothesis that moldboard plowing would have the greatest negative effect on soil quality indicators was verified. Based on this assessment, we recommend that to achieve and maintain good soil health, producers should strive to adopt less aggressive tillage practices.
\end{abstract}

Published by Elsevier B.V.

\section{Introduction}

Soil quality/health is a product of inherent (parent material, climate, and topography) and anthropogenic (tillage and crop rotation) interactions (Karlen et al., 1997). Tillage, which evolved as part of anthropogenic soil management, gradually became an integral part of crop production systems such that by 4000 years before present (BP), wooden plows drawn by oxen were used to scratch the soil surface in Europe, China, and many other regions. However, plows that actually inverted the soil were not developed

\footnotetext{
* Corresponding author. Tel.: +1 515294 3336; fax: +1 5152948125.

E-mail addresses: Doug.Karlen@ars.usda.gov (D.L. Karlen), Cindy.Cambardella@ars.usda.gov (C.A. Cambardella),

John.Kovar@ars.usda.gov (J.L. Kovar), oxfrdfrm@huxcomm.net (T.S. Colvin).

${ }^{1}$ Retired.
}

until the 17th century. European advancements during the 18th century resulted in the moldboard plow which turned the soil by $135^{\circ}$ and significantly improved weed control (Derpsch, 1998). This was of tremendous importance to many Europeans and especially the Germans because with soil inversion, they could overcome quackgrass (Agropyron repens) infestations that had become nearly impossible to control.

Presumably the European experience with moldboard plowing contributed to its widespread use when immigrants from those regions needed to break the prairie as they settled the U.S. Midwest (Karlen et al., 2010). Unfortunately by the mid 1930s, plowing had caused $40 \%$ of the land area in Iowa to lose $50-70 \%$ of its surface soil, which was often deposited in rivers and lakes as silt (Chase, 1936). Opportunities to slow this natural resource degradation by decreasing the intensity of tillage became scientifically feasible following World War II. Development of plant growth regulators (i.e., herbicides) and improved fertilizer management strategies 
made it feasible to grow crops with less and less tillage (Phillips and Phillips, 1984).

The need for information on less intensive tillage systems for Midwestern USA soils was a primary reason for initiating tillage and crop rotation studies at this research site in 1975 (Erbach, 1982). To quantify long-term effects of those practices after 31 years of known management (Karlen et al., 2013), an intensive sampling and soil quality indicator assessment was conducted to test our hypothes that intensive tillage practices were degrading soil quality/health.

To quantify long-term tillage effects, the Soil Management Assessment Framework (SMAF) developed by Andrews et al. (2004) was used as a soil quality assessment tool. The SMAF uses a 3-step process to assess soil quality. This includes indicator selection, indicator interpretation, and integration into an overall soil quality index or SQI. Soil taxonomy provides the foundation for each assessment based on soil suborder characteristics. Assessment values are generally expressed as a fraction or percentage of full performance for soil functions such as crop productivity, nutrient cycling, or environmental filtering and buffering. Currently, the SMAF has scoring curves or interpretation algorithms for 13 indicators: water-stable macroaggregation (WSA), plantavailable water (PAW), water-filled pore space (WFPS), bulk density (BD), electrical conductivity (EC), $\mathrm{pH}$, sodium adsorption ratio (SAR), extractable $\mathrm{P}$ and $\mathrm{K}$, soil organic carbon (SOC), microbial biomass carbon (MBC), potentially mineralizable $\mathrm{N}$ (PMN), and $\beta$-glucosidase (BG) activity (Andrews et al., 2004; Wienhold et al., 2009; Stott et al., 2010).

The SMAF has been used in the U.S. and abroad to evaluate nearsurface $(0-5$ and $5-15 \mathrm{~cm}$ ) soil properties and processes (e.g. Fernandez-Ugale et al., 2009; Imaz et al., 2010; Jokela et al., 2009; Karlen et al., 2006, 2011; Liebig et al., 2006; Stott et al., 2011; Wienhold et al., 2006; Zobeck et al., 2008). The unique aspect of this study is the longevity of the tillage and crop rotation treatments and the intensive analysis of soil samples representing two important texture groups (loam and clay loam). We hypothesized that intensive tillage (i.e., moldboard plowing) and acidification due to higher $\mathrm{N}$ fertilizer applications for continuous corn (CC) would have the most notable effects on soil quality. We also hypothesized that the differences would be more detectable within loam sampling sites because they are located at a slightly higher elevation $(\sim 2-3 \mathrm{~m})$ and are often more eroded than the clay loam sites. We tested these hypotheses by intensively sampling 40 , 0.3 ha plots where five tillage practices had been evaluated for $\mathrm{C} / \mathrm{S}$ or CC rotations for 31 or 26 years, respectively.

\section{Methods and materials}

\subsection{Site characteristics and general experimental design}

The long-term tillage and crop rotation study for which this soil quality/soil health assessment was conducted was initiated in 1975 (Erbach, 1982) at the Iowa State University (ISU) Agronomy/ Ag Engineering Research and Education Center (AAEREC) in Boone County, IA (latitude $42^{\circ} 01^{\prime} \mathrm{N}$, longitude $93^{\circ} 45^{\prime} \mathrm{W}$ ). As summarized by Karlen et al. (2013) there initially were eight "replicates" of each tillage system with four each managed in each phase of a corn and soybean rotation. Each tillage plot was $33 \mathrm{~m}$ wide and $91 \mathrm{~m}$ long except on the northwest and southeast corners of this 10-ha research site, where grass waterways (installed to capture and divert surface runoff from the research site) resulted in slightly shorter plots (Fig. 1). The tillage systems were: (1) slot plant on ridges which was eventually transitioned to a no-till operation; (2) spring disk; (3) till plant (ridge tillage) where surface soil to a depth of $\sim 5-8 \mathrm{~cm}$ is "thrown off" during planting and then the ridges are later rebuilt through cultivation; (4) fall moldboard plowing; and (5) fall chisel plowing. From 1976 through 1980, the plots were managed in an alternating corn and soybean rotation. Starting in 1979 and continuing through 2006, the four replicates in the south half of the site (Fig. 1) were used for continuous corn (CC) production, while the north half was maintained in the corn/ soybean $(\mathrm{C} / \mathrm{S})$ rotation with each crop occurring every other year.

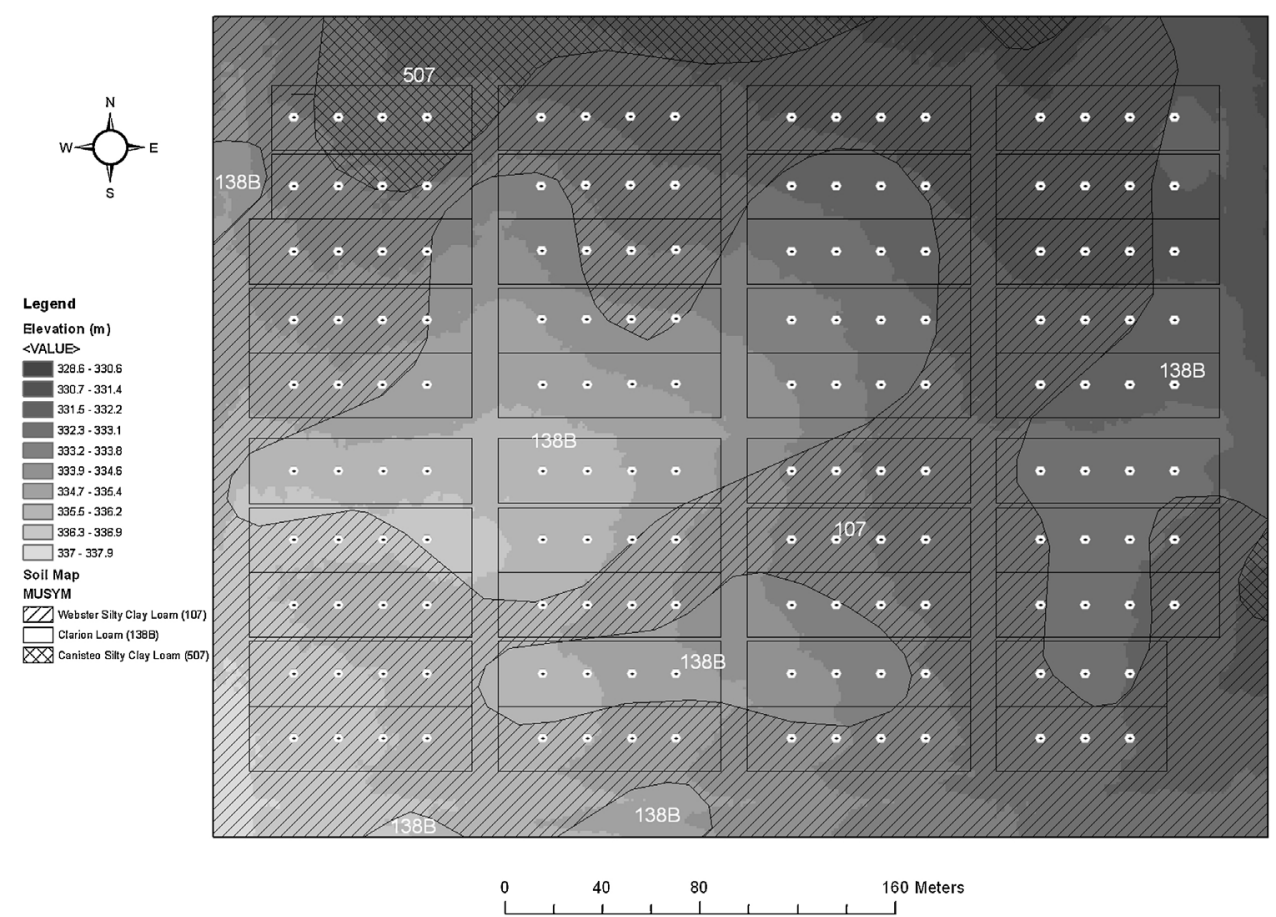

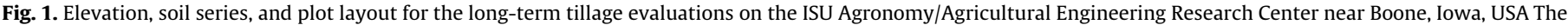

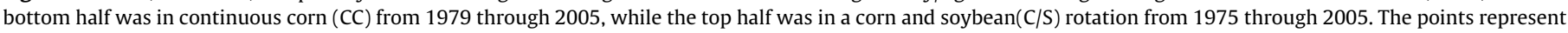
the 2005 soil sampling sites. 
Soils at this site are classified by the USDA Soil Survey Staff (2010) as Clarion loam (Fine-loamy, mixed, superactive, mesic Typic Hapludolls), Canisteo clay loam (Fine-loamy, mixed, superactive, calcareous, mesic Typic Endoaquolls), and Webster silty clay loam (Fine-loamy, mixed, superactive, mesic Typic Endoaquolls). Composite soil samples consisting of 12 soil cores were collected at four locations within each plot using a 32-mm diameter soil probe following the 2005 grain harvest. Soil cores were separated into two depth increments, $0-5 \mathrm{~cm}$ and $5-15 \mathrm{~cm}$ and combined by depth increment into four composite samples per plot. A fixed sampling pattern was used with each composite sample being taken within a $3 \mathrm{~m}$ diameter circle 18-, 37-, 49-, and 73-m from the west edge of each plot (Fig. 1). Both clay loam soil series were pooled into a single group for comparison with samples from loam sites. This approach resulted in 61 sampling sites classified as loam and 97 classified as clay loam.

\subsection{A summary of long-term agronomic practices}

Long-term soil and crop management records, crop yields, profile soil-test $\mathrm{P}$ and $\mathrm{K}$ and net returns for the five systems were summarized by Karlen et al. (2013). In general, all management decisions reflected best management practices associated with establishment (1975 to 1980), maintenance (1981 through 2002) and recovery/intensification (2003 through 2006) phases for this experiment. Commercially available corn and soybean cultivars were planted in $76 \mathrm{~cm}$ rows between late April and early June each year. Total fertilizer N-P-K application for the C/S rotation was 2956-423-852 $\mathrm{kg} \mathrm{ha}^{-1}$, respectively, while for the CC field, a total of 5022-280-508 $\mathrm{kg} \mathrm{ha}^{-1}$, respectively, was applied (Karlen et al., 2013).

\subsection{Soil quality assessment}

To assess long-term tillage and cropping system effects on soil quality, each composite soil sample was placed in a plastic bag, stored on ice until it could be transported to the lab, and then refrigerated at $4{ }^{\circ} \mathrm{C}$ until further processing. The field-moist samples were weighed and mixed before taking a $100 \mathrm{~g}$ subsample and drying it at $105{ }^{\circ} \mathrm{C}$ to determine soil water content. The total field-moist weight per composite sample was adjusted to a dry weight and divided by the volume associated with the 12 soil cores to estimate bulk density (BD) (Arshad et al., 1996; Blake and Hartge, 1986) for the $0-5-\mathrm{cm}$ and 5-15-cm depths. The remaining field-moist soil sample was passed through an 8-mm screen, airdried, and then crushed to pass a 2-mm sieve. Sub-samples from each depth increment were analyzed for soil $\mathrm{pH}$ (Watson and Brown, 1998) and electrical conductivity (EC) (Whitney, 1998) using a $1: 1$ soil to water ratio. A second sub-sample was extracted with $2 \mathrm{M} \mathrm{KCl}$ and analyzed for ammonium $\left(\mathrm{NH}_{4}-\mathrm{N}\right)$ and nitrate $\left(\mathrm{NO}_{3}-\mathrm{N}\right)$ concentrations using flow injection analysis (Lachat $\mathrm{QC}$ 800; Loveland, CO). A third set of sub-samples was submitted to a commercial soil-testing laboratory and analyzed for Bray-1 extractable P (Bray P) (Bray and Kurtz, 1945) and ammoniumacetate $\left(\mathrm{NH}_{4} \mathrm{OAc}\right)$ exchangeable (Ex-) $\mathrm{K}, \mathrm{Ca}, \mathrm{Mg}$ and Na concentrations (Warncke and Brown, 1998).

A fourth sub-sample was created by placing approximately $30 \mathrm{~g}$ of air-dry soil in a glass jar with steel rods, closing the jar and placing the sample on a roller belt for approximately $24 \mathrm{~h}$ in order to pulverize the soil before analyzing it for total carbon (TC) and total nitrogen (TN) by dry combustion. Based on soil $\mathrm{pH}$, neither depth increment appeared to have sufficient inorganic carbon (IC) to warrant volumetric measurement (Wagner et al., 1998), so TC and total organic carbon (TOC) were assumed to be the same.

Water-stable aggregation was determined using a modified Yoder (1936) wet-sieving apparatus designed to mechanically agitate a stack of five-sieves to separate macroaggregates into five size classes (4-8, 2-4, 1-2, 0.5-1 and 0.25-0.50 mm). A 100-g sample of air-dried, $8 \mathrm{~mm}$ sieved soil was placed on top of the stack of sieves and the sieve stack was agitated for 5 min at $125 \mathrm{rpm}$ with a vertical stroke length of $1.8 \mathrm{~cm}$. Wet aggregate stability (percent macro-aggregation) was calculated by summing aggregate mass for all classes greater than $0.25-\mathrm{mm}$, dividing by the total soil mass, and multiplying by 100 .

Microbial biomass $\mathrm{C}$ was measured by fumigation and direct extraction with $0.5 \mathrm{M} \mathrm{K}_{2} \mathrm{SO}_{4}$ on 8-mm-sieved field-moist samples (Vance et al., 1987). Organic $C$ in the fumigated and non-fumigated extracts was measured using a Dohrmann DC-180 carbon analyzer (Rosemount Analytical Services, Santa Clara, CA) and biomass C was calculated using the correction factor $(k=0.33)$ of Sparling and West (1988). Particulate organic matter (POM) was determined as described by Cambardella and Elliott (1992). Nitrogen mineralization potential was quantified after 28-day aerobic incubation using a modification of Keeney and Bremner's (1967) method as described by Drinkwater et al. (1996).

\subsection{Statistical analyses}

The data were analyzed by cropping system and depth increment using a SAS General Linear Model (GLM) to determine tillage system, textural group, replicate and tillage system by textural group interactions (SAS Institute, 1990). Least significant difference (LSD) values were calculated to separate means for factors that had $F$ values of $P \leq 0.10$. A $10 \%$ significance level was chosen because inherent variability that can be blocked in small replicated plot studies often masks field-scale differences important to land managers. Mean values for nine of the indicators (Tables 1 and 2) were used for SMAF analyses to determine how tillage affected soil quality for the two textural groups and crop rotations. Physical effects are represented by WSA and BD, while chemical effects are represented by $\mathrm{pH}, \mathrm{EC}$, Bray-P and Ex-K, and biological effects by TOC, MBC, and PMN. This approach is consistent with the general SMAF guidelines which recommend using a minimum of five indicators with at least one each representing anticipated soil biological, chemical, and physical property or process responses (Karlen et al., 2008). The indicators were scored by transforming mean measured values into $0-1$ values using the SMAF scoring curves. Those curves account for organic matter, texture, climate, slope, region, mineralogy, weathering class, crop, sampling time, and analytical method effects on the various threshold values (Andrews et al., 2004; Wienhold et al., 2009; Stott et al., 2010). The indicator scores were examined individually and as an overall soil quality index (SQI) to determine how the nine parameters were affected by tillage, crop rotation, and soil series. No additional statistical analyses were run on the SMAF scores since they were calculated using mean values for the selected indicators.

\section{Results and discussion}

\subsection{Tillage system differences for SMAF indicator means}

Measurements of WSA at both depth increments (Table 1) showed lower values for the moldboard plow treatment indicating a less stable soil condition and a surface that would be more vulnerable to wind and water erosion. Differences in BD within the 0-5-cm increment were small but the moldboard plow treatment did have significantly higher values in both fields, presumably because the number of tillage operations (i.e., plowing, 1-3 disk operations, and 1-2 field cultivations) resulted in greater break up, incorporation, and oxidation of above- and below-ground plant residues during the course of this study. Therefore from a physical 
Table 1

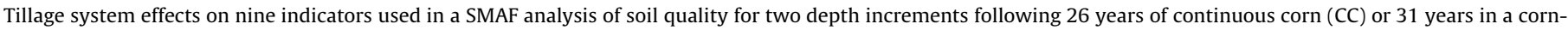
soybean $(C / S)$ rotation on glacial till soils in central Iowa.

\begin{tabular}{|c|c|c|c|c|c|c|c|c|c|}
\hline Tillage system & $\begin{array}{l}W_{S A}^{a} \\
\left(\mathrm{~g} \mathrm{~kg}^{-1}\right)\end{array}$ & $\begin{array}{l}\mathrm{BD} \\
\left(\mathrm{g} \mathrm{cm}^{-3}\right)\end{array}$ & $\mathrm{pH}$ & $\begin{array}{l}\mathrm{EC} \\
\left(\mu \mathrm{scm}^{-1}\right)\end{array}$ & $\begin{array}{l}\text { Bray P } \\
\left(\mathrm{mg} \mathrm{kg}^{-1}\right)\end{array}$ & $\begin{array}{l}\mathrm{Ex}-\mathrm{K} \\
\left(\mathrm{mg} \mathrm{kg}^{-1}\right)\end{array}$ & $\begin{array}{l}\text { TOC } \\
\left(\mathrm{g} \mathrm{kg}^{-1}\right)\end{array}$ & $\begin{array}{l}\text { MBC } \\
\left(\mu g g^{-1}\right)\end{array}$ & $\begin{array}{l}\text { PMN } \\
\left(\mathrm{mgg} \mathrm{g}^{-1}\right)\end{array}$ \\
\hline & \multicolumn{9}{|c|}{ CC: $0-5 \mathrm{~cm}$ depth } \\
\hline Moldboard plow & 298 & 1.2 & 6.5 & 102 & 17 & 110 & 20 & 179 & 26 \\
\hline Chisel plow & 383 & 1.1 & 6.4 & 106 & 39 & 131 & 25 & 302 & 52 \\
\hline Spring disk & 410 & 1.0 & 6.8 & 111 & 42 & 162 & 28 & 481 & 54 \\
\hline Ridge-tillage & 456 & 1.1 & 6.4 & 112 & 35 & 135 & 29 & 466 & 66 \\
\hline No-tillage & 483 & 1.0 & 6.6 & 109 & 36 & 171 & 30 & 504 & 71 \\
\hline \multirow[t]{2}{*}{$\operatorname{LSD}_{(0.1)}$} & 31 & 0.1 & 0.2 & 4 & 6 & 17 & 2 & 61 & 12 \\
\hline & \multicolumn{9}{|c|}{ CC: $5-15 \mathrm{~cm}$ depth } \\
\hline Moldboard plow & 352 & 1.2 & 6.7 & 103 & 15 & 79 & 20 & 147 & 29 \\
\hline Chisel plow & 443 & 1.2 & 6.4 & 102 & 30 & 84 & 24 & 178 & 35 \\
\hline Spring disk & 448 & 1.1 & 6.7 & 104 & 27 & 100 & 24 & 247 & 37 \\
\hline Ridge-tillage & 447 & 1.2 & 6.4 & 101 & 25 & 84 & 22 & 194 & 32 \\
\hline No-tillage & 489 & 1.1 & 6.8 & 101 & 25 & 95 & 24 & 240 & 37 \\
\hline \multirow[t]{2}{*}{$\operatorname{LSD}_{(0.1)}$} & 39 & $\mathrm{NS}^{\mathrm{b}}$ & 0.2 & 2 & 5 & 11 & 2 & 42 & 6 \\
\hline & \multicolumn{9}{|c|}{ C/S: $0-5 \mathrm{~cm}$ depth } \\
\hline Moldboard plow & 280 & 1.3 & 6.4 & 104 & 19 & 110 & 21 & 247 & 20 \\
\hline Chisel plow & 307 & 1.0 & 6.1 & 102 & 28 & 124 & 25 & 359 & 43 \\
\hline Spring disk & 382 & 1.0 & 6.2 & 106 & 33 & 131 & 28 & 410 & 42 \\
\hline Ridge-tillage & 352 & 1.1 & 6.5 & 109 & 37 & 130 & 27 & 412 & 54 \\
\hline No-tillage & 386 & 1.0 & 6.3 & 104 & 35 & 127 & 28 & 495 & 47 \\
\hline \multirow[t]{2}{*}{$\operatorname{LSD}_{(0.1)}$} & 33 & 0.1 & 0.2 & 3 & 5 & 15 & 2 & 53 & 9 \\
\hline & \multicolumn{9}{|c|}{ C/S: $5-15 \mathrm{~cm}$ depth } \\
\hline Moldboard plow & 330 & 1.1 & 6.5 & 101 & 18 & 98 & 21 & 217 & 24 \\
\hline Chisel plow & 363 & 1.1 & 6.1 & 101 & 22 & 101 & 23 & 247 & 28 \\
\hline Spring disk & 426 & 1.1 & 6.1 & 102 & 23 & 97 & 24 & 258 & 27 \\
\hline Ridge-tillage & 382 & 1.1 & 6.1 & 101 & 25 & 102 & 23 & 186 & 24 \\
\hline No-tillage & 410 & 1.1 & 6.4 & 102 & 25 & 93 & 22 & 265 & 23 \\
\hline $\operatorname{LSD}_{(0.1)}$ & 48 & NS & 0.2 & NS & 5 & 10 & 2 & 38 & NS \\
\hline
\end{tabular}

a WSA: water stable macroaggregate fraction; BD: bulk density; $\mathrm{pH}$ : acidity/alkalinity; EC: electrical conductivity; Bray P: extracatable P using the Bray P1 reagent; Ex-K: $\mathrm{NH}_{4} \mathrm{OAc}$ exchangeable K; TOC: total organic carbon; MBC: microbial biomass carbon; PMN: potentially mineralizable nitrogen.

b NS: indicates no statistical significance among mean values at $P \leq 0.1$.

Table 2

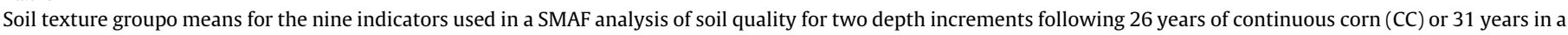
corn-soybean $(\mathrm{C} / \mathrm{S})$ rotation on glacial till derived soils in central Iowa.

\begin{tabular}{|c|c|c|c|c|c|c|c|c|c|}
\hline Texture group & $\begin{array}{l}W_{S A}^{a} \\
\left(\mathrm{~g} \mathrm{~kg}^{-1}\right)\end{array}$ & $\begin{array}{l}\mathrm{BD} \\
\left(\mathrm{g} \mathrm{cm}^{-3}\right)\end{array}$ & $\mathrm{pH}$ & $\begin{array}{l}\mathrm{EC} \\
\left(\mu \mathrm{sm}^{-1}\right)\end{array}$ & $\begin{array}{l}\text { Bray P } \\
\left(\mathrm{mg} \mathrm{kg}^{-1}\right)\end{array}$ & $\begin{array}{l}\text { Ex-K } \\
\left(\mathrm{mg} \mathrm{kg}^{-1}\right)\end{array}$ & $\begin{array}{l}\text { TOC } \\
\left(\mathrm{g} \mathrm{kg}^{-1}\right)\end{array}$ & $\begin{array}{l}\mathrm{MBC} \\
\left(\mu \mathrm{gg}^{-1}\right)\end{array}$ & $\begin{array}{l}\text { PMN } \\
\left(\mathrm{mg} \mathrm{g}^{-1}\right)\end{array}$ \\
\hline & \multicolumn{9}{|c|}{ CC: $0-5 \mathrm{~cm}$ depth } \\
\hline Loam & 389 & 1.1 & 6.7 & 106 & 26 & 140 & 24 & 347 & 56 \\
\hline Clay loam & 418 & 1.0 & 6.4 & 109 & 39 & 144 & 28 & 414 & 53 \\
\hline \multirow[t]{2}{*}{$\operatorname{LSD}_{(0.1)}$} & 20 & 0.1 & 0.2 & 3 & 4 & $\mathrm{NS}^{\mathrm{b}}$ & 1 & 40 & NS \\
\hline & \multicolumn{9}{|c|}{ CC: $5-15 \mathrm{~cm}$ depth } \\
\hline Loam & 418 & 1.1 & 6.7 & 102 & 19 & 86 & 21 & 185 & 34 \\
\hline Clay loam & 448 & 1.2 & 6.5 & 102 & 28 & 90 & 24 & 213 & 34 \\
\hline \multirow[t]{2}{*}{$\operatorname{LSD}_{(0.1)}$} & 25 & NS & 0.2 & NS & 3 & NS & 1 & 27 & NS \\
\hline & \multicolumn{9}{|c|}{ C/S: $0-5 \mathrm{~cm}$ depth } \\
\hline Loam & 296 & 1.2 & 6.4 & 104 & 23 & 110 & 21 & 302 & 43 \\
\hline Clay loam & 369 & 1.0 & 6.3 & 105 & 35 & 133 & 28 & 434 & 41 \\
\hline \multirow[t]{2}{*}{$\operatorname{LSD}_{(0.1)}$} & 22 & 0.1 & NS & NS & 3 & 10 & 2 & 34 & NS \\
\hline & \multicolumn{9}{|c|}{ C/S: $5-15 \mathrm{~cm}$ depth } \\
\hline Loam & 314 & 1.1 & 6.2 & 101 & 18 & 100 & 19 & 188 & 25 \\
\hline Clay loam & 423 & 1.1 & 6.3 & 102 & 26 & 98 & 24 & 263 & 25 \\
\hline $\operatorname{LSD}_{(0.1)}$ & 31 & 0.03 & NS & NS & 3 & NS & 1 & 25 & NS \\
\hline
\end{tabular}

a WSA: water stable macroaggregate fraction; BD: bulk density; pH: acidity/alkalinity; EC: electrical conductivity; Bray P: extracatable P using the Bray P1 reagent; Ex-K: $\mathrm{NH}_{4} \mathrm{OAc}$ exchangeable K; TOC: total organic carbon; MBC: microbial biomass carbon; PMN: potentially mineralizable nitrogen.

b NS: indicates no statistical significance among mean values at $P \leq 0.1$.

soil quality indicator perspective, both WSA and BD indicate that the more aggressive tillage practices had a negative effect.

Among the chemical indicators (Table 1 ) of soil quality ( $\mathrm{pH}, \mathrm{EC}$, Bray-P and Ex-K) there were statistical differences among tillage treatments for $\mathrm{pH}$ and $\mathrm{EC}$, but differences among means were small and would have virtually no effect on crop emergence, growth or productivity. Bray-P was significantly lower in both fields and at both depth increments in the moldboard plow treatments and except for the 5-15-cm depth increment Ex-K was also significantly lower after more than 30 years of moldboard plowing. Based on ISU soil test interpretations (Sawyer et al., 2011), the Bray-P values were still in the optimum range for all tillage treatments, but as discussed by Karlen et al. (2013) Ex-K levels were low and perhaps yield limiting.

Examining the carbon-based biological indicators (Table 1), we see that at both the $0-5-$ and 5-15-cm sampling depths, 
moldboard plowing resulted in the lowest TOC concentrations after 26 years of $C C$ or 31 years in a $C / S$ rotation. The two microbialmediated indicators (MBC and PMN) also show the lowest values at both sampling depth for the moldboard plowing under CC and at the $0-5-\mathrm{cm}$ depth in the $\mathrm{C} / \mathrm{S}$ field. Some may argue that since only the $0-5$ - and $5-15-\mathrm{cm}$ depth increments were analyzed, it is possible that lower TOC, MBC, and PMN values at those depths simply reflects burying of crop residue below $15 \mathrm{~cm}$ through the moldboard plowing operation. However, based on similar studies by Varvel and Wilhelm (2011) that showed greater soil N and SOC levels deep in the profile beneath less aggressive tillage practices and unpublished profile carbon data from this site, we conclude that a substantial portion of the carbon added annually through photosynthesis was lost when tillage practices were aggressive.

Data in Table 1 also show a stratification effect for all three biological indicators evident when comparing sampling depths. Averaging measurements for the two fields shows mean values of 26 vs. $23 \mathrm{~g} \mathrm{~kg}^{-1}$ for TOC, 386 vs. $218 \mu \mathrm{g} \mathrm{g}^{-1}$ for MBC, and $48 \mathrm{vs.}$ $30 \mathrm{mg} \mathrm{g}^{-1}$ for PMN within the $0-5-$ and $5-15-\mathrm{cm}$ increments, respectively. Furthermore, within both the CC and C/S fields, all three indicators are higher in long-term no-tillage treatments than all other tillage treatments in the $0-5-\mathrm{cm}$ increment, but among the lowest values within the $5-15-\mathrm{cm}$ increment.

\subsection{Soil texture group differences among SMAF indicator means}

Mean surface soil quality indicator values for the two soil texture groups within both fields are presented in Table 2. For WSA, significant differences were found in both fields and at both depth increments, with the more sloping, loam sites having lower WSA values than the relatively flat, clay loam sites. Texture is an obvious contributing factor to these differences, but of more importance for long-term soil security, lower values for loam soils, which are located at a slightly higher elevation, mean they are also more vulnerable to wind and water erosion. A comparison between the two fields shows that CC increased WSA at both depth increments and in both texture groups when compared to the adjacent C/S field. Presumably this also reflected greater carbon inputs with CC, as soybean is known to be a "low residue" crop and thus more prone to creating more erosive soil conditions (Alberts et al., 1985). For BD, there were statistically significant differences between the two soil texture groups, but they were very small and would have no measureable impact on productivity of either crop.

Among the chemical indicators of soil quality/health (Table 2), Bray $\mathrm{P}$ showed the most consistent differences due to textural group with the loam sites being lower than the clay loam sites. However, the concentrations at both depths were sufficient for good corn and soybean production. Ex-K showed significant differences between the two textural groups within the $\mathrm{C} / \mathrm{S}$ field, but more notably for the site was that except for the loam sites in the CC field, all Ex-K levels were low to very low based on ISU soiltest guidelines (Sawyer et al., 2011). Soil pH and EC showed few significant differences between texture groups, with none that would be expected to affect crop growth and development.

Among biological soil quality indicators, TOC and MBC concentrations were significantly lower in the loam samples than in clay loam soils (Table 2), but PMN levels showed no significant differences between soil texture groups. This was a bit unexpected since $\mathrm{C}$ and $\mathrm{N}$ pools often show similar patterns. We attribute this to variability and perhaps the presence of $\mathrm{N}$ not associated with the TOC or MBC fractions.

\subsection{SMAF analysis of tillage system and SMU effects}

The SMAF scores for each of the nine indicators as well as an overall SQI for comparing the five tillage systems and two soil texture groups within the long-term CC and C/S fields are presented in Tables 3 and 4, respectively. Those data show that

Table 3

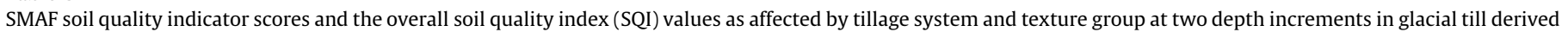
soils after 26 years of continuous corn (CC) in central Iowa.

\begin{tabular}{|c|c|c|c|c|c|c|c|c|c|c|c|}
\hline Tillage & Texture group & $\mathrm{WSA}^{\mathrm{a}}$ & $\mathrm{BD}$ & $\mathrm{pH}$ & $\mathrm{EC}$ & Bray P & Ex-K & TOC & MBC & PMN & SQI \\
\hline & \multicolumn{11}{|c|}{$0-5 \mathrm{~cm}$} \\
\hline Plow & Loam & 0.96 & 0.99 & 0.98 & 0.62 & 1.00 & 0.79 & 0.67 & 0.98 & 1.00 & 0.89 \\
\hline Chisel & Loam & 0.90 & 0.99 & 1.00 & 0.61 & 0.99 & 0.75 & 0.53 & 0.62 & 1.00 & 0.82 \\
\hline Disk & Loam & 0.90 & 0.99 & 0.99 & 0.65 & 1.00 & 0.85 & 0.74 & 0.98 & 1.00 & 0.90 \\
\hline Ridge-till & Loam & 0.94 & 0.99 & 1.00 & 0.65 & 1.00 & 0.78 & 0.84 & 0.99 & 1.00 & 0.91 \\
\hline No-till & Loam & 0.71 & 0.99 & 1.00 & 0.59 & 0.97 & 0.69 & 0.45 & 0.31 & 0.94 & 0.74 \\
\hline Average & & 0.88 & 0.99 & 0.99 & 0.62 & 0.99 & 0.77 & 0.65 & 0.77 & 0.99 & 0.85 \\
\hline Plow & Clay loam & 0.94 & 0.99 & 1.00 & 0.66 & 1.00 & 1.00 & 0.82 & 0.97 & 1.00 & 0.93 \\
\hline Chisel & Clay loam & 0.79 & 0.96 & 1.00 & 0.62 & 1.00 & 0.89 & 0.55 & 0.63 & 1.00 & 0.83 \\
\hline Disk & Clay loam & 0.85 & 0.99 & 1.00 & 0.66 & 1.00 & 0.93 & 0.65 & 0.96 & 1.00 & 0.89 \\
\hline Ridge-till & Clay loam & 0.90 & 0.99 & 1.00 & 0.66 & 1.00 & 0.90 & 0.65 & 0.90 & 1.00 & 0.89 \\
\hline No-till & Clay loam & 0.66 & 0.97 & 1.00 & 0.61 & 0.98 & 0.83 & 0.32 & 0.22 & 0.87 & 0.72 \\
\hline \multirow[t]{2}{*}{ Average } & & 0.83 & 0.98 & 1.00 & 0.64 & 1.00 & 0.91 & 0.60 & 0.74 & 0.97 & 0.85 \\
\hline & $5-15 \mathrm{~cm}$ & & & & & & & & & & \\
\hline Plow & Loam & 0.93 & 0.99 & 0.98 & 0.59 & 0.98 & 0.56 & 0.38 & 0.33 & 0.99 & 0.75 \\
\hline Chisel & Loam & 0.83 & 0.99 & 1.00 & 0.60 & 0.97 & 0.56 & 0.42 & 0.20 & 1.00 & 0.73 \\
\hline Disk & Loam & 0.94 & 0.99 & 0.99 & 0.61 & 1.00 & 0.68 & 0.60 & 0.66 & 1.00 & 0.83 \\
\hline Ridge-till & Loam & 0.97 & 0.99 & 1.00 & 0.59 & 1.00 & 0.55 & 0.67 & 0.23 & 0.99 & 0.78 \\
\hline No-till & Loam & 0.93 & 0.99 & 1.00 & 0.61 & 0.96 & 0.56 & 0.44 & 0.24 & 0.96 & 0.74 \\
\hline Average & & 0.92 & 0.99 & 0.99 & 0.60 & 0.98 & 0.58 & 0.50 & 0.33 & 0.99 & 0.77 \\
\hline Plow & Clay loam & 1.00 & 0.96 & 1.00 & 0.59 & 1.00 & 0.83 & 0.64 & 0.55 & 1.00 & 0.84 \\
\hline Chisel & Clay loam & 0.73 & 0.87 & 1.00 & 0.60 & 1.00 & 0.74 & 0.54 & 0.24 & 0.99 & 0.75 \\
\hline Disk & Clay loam & 0.90 & 0.89 & 1.00 & 0.61 & 1.00 & 0.75 & 0.52 & 0.34 & 0.99 & 0.78 \\
\hline Ridge-till & Clay loam & 0.88 & 0.85 & 1.00 & 0.59 & 1.00 & 0.73 & 0.38 & 0.24 & 0.97 & 0.74 \\
\hline No-till & Clay loam & 0.89 & 0.87 & 1.00 & 0.60 & 0.96 & 0.71 & 0.31 & 0.14 & 0.95 & 0.71 \\
\hline Average & & 0.88 & 0.89 & 1.00 & 0.60 & 0.99 & 0.75 & 0.48 & 0.30 & 0.98 & 0.76 \\
\hline
\end{tabular}

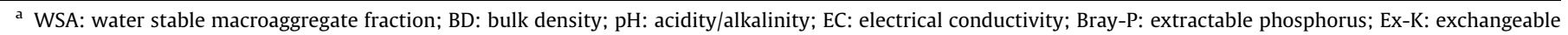
potassium; TOC: total organic carbon; MBC: microbial biomass carbon; PMN: potentially mineralizable nitrogen; SQI: soil quality index. 
Table 4

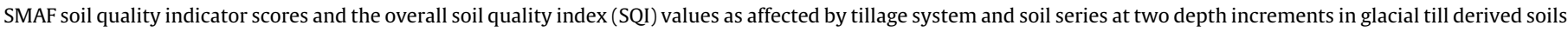
after 31 years of a corn-soybean rotation $(C / S)$ in central Iowa.

\begin{tabular}{|c|c|c|c|c|c|c|c|c|c|c|c|}
\hline Tillage & Texture group & WSA $^{\mathrm{a}}$ & $\mathrm{BD}$ & $\mathrm{pH}$ & EC & Bray P & Ex-K & TOC & $\mathrm{MBC}$ & PMN & SQI \\
\hline Plow & Loam & 0.81 & 0.99 & 1.00 & 0.61 & 0.99 & 0.70 & 0.55 & 0.93 & 1.00 & 0.84 \\
\hline Chisel & Loam & 0.71 & 0.99 & 1.00 & 0.60 & 1.00 & 0.70 & 0.52 & 0.86 & 1.00 & 0.82 \\
\hline Disk & Loam & 0.71 & 0.99 & 1.00 & 0.63 & 1.00 & 0.69 & 0.60 & 0.82 & 1.00 & 0.83 \\
\hline Ridge-till & Loam & 0.81 & 0.99 & 1.00 & 0.64 & 1.00 & 0.69 & 0.52 & 0.81 & 1.00 & 0.83 \\
\hline No-till & Loam & 0.64 & 0.95 & 1.00 & 0.59 & 0.97 & 0.69 & 0.42 & 0.59 & 0.82 & 0.74 \\
\hline Average & & 0.74 & 0.98 & 1.00 & 0.61 & 0.99 & 0.69 & 0.52 & 0.80 & 0.96 & 0.81 \\
\hline Plow & Clay loam & 0.84 & 0.99 & 1.00 & 0.61 & 1.00 & 0.90 & 0.68 & 0.97 & 1.00 & 0.89 \\
\hline Chisel & Clay loam & 0.72 & 0.99 & 1.00 & 0.61 & 1.00 & 0.91 & 0.63 & 0.82 & 0.99 & 0.85 \\
\hline Disk & Clay loam & 0.84 & 0.99 & 1.00 & 0.62 & 1.00 & 0.90 & 0.63 & 0.86 & 1.00 & 0.87 \\
\hline Ridge-till & Clay loam & 0.90 & 0.99 & 1.00 & 0.64 & 1.00 & 0.91 & 0.69 & 0.92 & 1.00 & 0.90 \\
\hline No-till & Clay loam & 0.63 & 0.53 & 1.00 & 0.62 & 0.98 & 0.84 & 0.36 & 0.39 & 0.51 & 0.65 \\
\hline Average & & 0.78 & 0.90 & 1.00 & 0.62 & 1.00 & 0.89 & 0.60 & 0.79 & 0.90 & 0.83 \\
\hline Plow & Loam & 0.83 & 0.99 & 1.00 & 0.60 & 0.97 & 0.65 & 0.37 & 0.33 & 0.58 & 0.70 \\
\hline Chisel & Loam & 0.78 & 0.99 & 1.00 & 0.59 & 0.98 & 0.65 & 0.42 & 0.48 & 0.99 & 0.76 \\
\hline Disk & Loam & 0.66 & 0.99 & 1.00 & 0.59 & 0.97 & 0.67 & 0.43 & 0.34 & 0.82 & 0.72 \\
\hline Ridge-till & Loam & 0.83 & 0.99 & 1.00 & 0.59 & 0.97 & 0.68 & 0.37 & 0.24 & 0.77 & 0.71 \\
\hline No-till & Loam & 0.71 & 0.99 & 1.00 & 0.59 & 0.97 & 0.65 & 0.44 & 0.47 & 0.92 & 0.75 \\
\hline Average & & 0.76 & 0.99 & 1.00 & 0.59 & 0.97 & 0.66 & 0.41 & 0.37 & 0.81 & 0.73 \\
\hline Plow & Clay loam & 0.88 & 0.96 & 1.00 & 0.60 & 1.00 & 0.75 & 0.46 & 0.54 & 0.84 & 0.78 \\
\hline Chisel & Clay loam & 0.85 & 0.96 & 1.00 & 0.59 & 1.00 & 0.82 & 0.55 & 0.27 & 0.80 & 0.76 \\
\hline Disk & Clay loam & 0.90 & 0.95 & 1.00 & 0.60 & 1.00 & 0.78 & 0.49 & 0.46 & 0.92 & 0.79 \\
\hline Ridge-till & Clay loam & 0.82 & 0.99 & 1.00 & 0.59 & 1.00 & 0.80 & 0.59 & 0.26 & 0.87 & 0.76 \\
\hline No-till & Clay loam & 0.77 & 0.94 & 1.00 & 0.59 & 0.98 & 0.78 & 0.38 & 0.30 & 0.75 & 0.72 \\
\hline Average & & 0.84 & 0.96 & 1.00 & 0.60 & 1.00 & 0.79 & 0.47 & 0.37 & 0.84 & 0.76 \\
\hline
\end{tabular}

a WSA: water stable macroaggregate fraction; BD: bulk density; pH: acidity/alkalinity; EC: electrical conductivity; Bray-P: extractable phosphorus; Ex-K: exchangeable potassium; TOC: total organic carbon; MBC: microbial biomass carbon; PMN: potentially mineralizable nitrogen; SQI: soil quality index.

following 26 years of $\mathrm{CC}$, the PMN, pH, and Bray $\mathrm{P}$ values all achieved SMAF scores of 1.0 (Table 3 ) indicating that for crop productivity, they were functioning at their full potential within both depth increments. Bulk density scores were also approximately 1.0 indicating no root-limiting conditions, except for the $5-15 \mathrm{~cm}$ depth within the clay loam sites where the average score was 0.89 . That would indicate a slight $(\sim 10 \%)$ reduction from full potential for crop production in those areas. WSA scores were generally the next lowest, ranging from 0.83 to 0.92 (i.e., $83-92 \%$ of full potential) and being about 5\% higher in the $5-15 \mathrm{~cm}$ increment for both soil texture groups. EC scores for all five tillage systems, both texture groups, and both sampling depths were all between 0.59 and 0.66 . These chemical soil quality indicator scores were somewhat lower than expected for Midwestern soils, but probably reflected high $\mathrm{Ca}$ and $\mathrm{Mg}$ concentrations as discussed in section 3.4. The Ex-K scores after long-term CC reflect low concentrations in the $5-15 \mathrm{~cm}$ increment as discussed previously (Tables 1 and 2) and were lowest for the more sloping Clarion loam than clay loam sites. The final two biological indicators of soil quality/health, TOC and MBC had the lowest SMAF scores in the CC field, especially within the 5 to $15 \mathrm{~cm}$ depth increment due to an apparent depletion of soil organic matter when compared to what is expected based on the soil-forming factors and inherent condition (USDA-SCS, 1981) of soils in this area.

The SMAF scores for samples collected following a 31-year C/S rotation are shown in Table 4 . Soil $\mathrm{pH}$, Bray $\mathrm{P}$, and BD scores for the five tillage systems, both texture groups and both depth increments were generally between 0.9 and 1.0 in this field indicating those factors were fully functioning with regard to crop production. The PMN indicator in the $0-5 \mathrm{~cm}$ depth increment also averaged 0.90 and 0.96 for the loam and clay loam sampling sites, respectively, but within the $5-15 \mathrm{~cm}$ depth increment, the average scores for that biological indicator decreased to 0.81 and 0.84 , respectively, indicating less potentially available $\mathrm{N}$ than would normally be expected at that depth for these soils. WSA and Ex-K scores were the next lowest at this site, ranging from 0.74 to 0.84 or 0.66 to 0.89 , respectively. For both of these indicators, average scores for the five tillage systems within clay loam sites were slightly higher than at loam sites, presumably reflecting the higher clay content that would affect both aggregate stability (WSA) and cation exchange capacity (CEC). As discussed for the CC field, EC scores once again averaged 0.6 for both texture groups and depth increments, presumably reflecting the high $\mathrm{Ca}$ and $\mathrm{Mg}$ concentrations in these soils. Also, as noted for the CC field, the final two biological soil quality indicators, TOC and MBC had the lowest SMAF scores with averages of 0.56 and 0.44 or 0.80 and 0.37 within the $0-5$ - and 5-15-cm depth increments, respectively. Again, we suggest these low scores reflect a depletion of soil organic matter when compared to inherent levels expected for these soils.

The average SQI for the five tillage systems within the $0-5 \mathrm{~cm}$ sampling depth for both texture groups in the CC field (Table 3) was 0.85 , indicating soils at that depth were functioning at $85 \%$ of their potential, but within the $5-15 \mathrm{~cm}$ depth increment, both texture groups were functioning at just over $75 \%$ of their potential. The lower SQI value for the $5-15 \mathrm{~cm}$ depth increment was primarily due to very low TOC and MBC scores, although Ex-K and BD scores were also lower at that depth. Within the $\mathrm{C} / \mathrm{S}$ field (Table 4), the average SQI for the five tillage treatments and two soil texture groups showed that following 31 years of a $C / S$ rotation, the loam and clay loam soils were functioning at 82 and $74 \%$ of their anticipated potential for crop production within the $0-$ 5- and 5-15-cm depth increments, respectively.

\subsection{Non-SMAF indicator evaluations}

In addition to the nine SMAF indicators that were measured and evaluated, 14 other soil measurements were made on samples from both depth increments at all 158 sites (Fig. 1). Those analyses 
Table 5

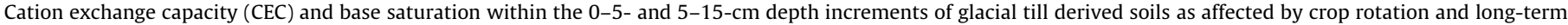
tillage treatments in central Iowa after 26 years of continuous corn (CC) or 31 years of a corn-soybean (C/S) rotation in central Iowa.

\begin{tabular}{|c|c|c|c|c|c|c|}
\hline \multirow[t]{2}{*}{ Tillage } & \multirow{2}{*}{$\begin{array}{l}\text { CEC } \\
\left(\mathrm{cmol} \mathrm{kg}^{-1}\right)\end{array}$} & \multicolumn{5}{|c|}{ Base saturation } \\
\hline & & $\begin{array}{l}\text { Total } \\
\left(\mathrm{g} \mathrm{kg}^{-1}\right)\end{array}$ & $\begin{array}{l}\mathrm{K} \\
\left(\mathrm{g} \mathrm{kg}^{-1}\right)\end{array}$ & $\begin{array}{l}\mathrm{Ca} \\
\left(\mathrm{g} \mathrm{kg}^{-1}\right)\end{array}$ & $\begin{array}{l}\mathrm{Mg} \\
\left(\mathrm{g} \mathrm{kg}^{-1}\right)\end{array}$ & $\begin{array}{l}\mathrm{H} \\
\left(\mathrm{g} \mathrm{kg}^{-1}\right)\end{array}$ \\
\hline & \multicolumn{6}{|c|}{ CC: $0-5 \mathrm{~cm}$ depth } \\
\hline Moldboard plow & 18 & 902 & 15 & 702 & 184 & 98 \\
\hline Chisel plow & 20 & 889 & 17 & 699 & 173 & 111 \\
\hline Spring disk & 19 & 985 & 22 & 812 & 150 & 15 \\
\hline Ridge-tillage & 18 & 889 & 19 & 690 & 180 & 111 \\
\hline No-tillage & 19 & 902 & 24 & 692 & 186 & 98 \\
\hline \multirow[t]{2}{*}{$\operatorname{LSD}_{(0.1)}$} & $\mathrm{NS}^{\mathrm{a}}$ & 66 & 3 & 57 & 18 & 66 \\
\hline & \multicolumn{6}{|c|}{ CC: $5-15 \mathrm{~cm}$ depth } \\
\hline Moldboard plow & 16 & 940 & 12 & 727 & 201 & 60 \\
\hline Chisel plow & 19 & 875 & 12 & 698 & 165 & 125 \\
\hline Spring disk & 17 & 961 & 15 & 781 & 165 & 39 \\
\hline Ridge-tillage & 20 & 859 & 11 & 671 & 176 & 141 \\
\hline No-tillage & 18 & 950 & 14 & 754 & 182 & 50 \\
\hline \multirow[t]{2}{*}{$\operatorname{LSD}_{(0.1)}$} & 2 & 64 & 2 & 50 & 22 & 64 \\
\hline & \multicolumn{6}{|c|}{ C/S: $0-5 \mathrm{~cm}$ depth } \\
\hline Moldboard plow & 17 & 904 & 16 & 702 & 186 & 96 \\
\hline Chisel plow & 20 & 832 & 17 & 692 & 174 & 167 \\
\hline Spring disk & 20 & 847 & 16 & 652 & 178 & 153 \\
\hline Ridge-tillage & 18 & 879 & 18 & 682 & 179 & 121 \\
\hline No-tillage & 19 & 866 & 17 & 658 & 191 & 134 \\
\hline \multirow[t]{2}{*}{$\operatorname{LSD}_{(0.1)}$} & 2 & 56 & 2 & 49 & 18 & 56 \\
\hline & \multicolumn{6}{|c|}{ C/S: $5-15 \mathrm{~cm}$ depth } \\
\hline Moldboard plow & 16 & 926 & 16 & 722 & 188 & 74 \\
\hline Chisel plow & 18 & 839 & 14 & 664 & 161 & 161 \\
\hline Spring disk & 19 & 833 & 14 & 641 & 178 & 167 \\
\hline Ridge-tillage & 19 & 812 & 14 & 639 & 158 & 188 \\
\hline No-tillage & 18 & 864 & 13 & 681 & 170 & 136 \\
\hline $\operatorname{LSD}_{(0.1)}$ & 2 & 70 & 2 & 55 & 20 & 70 \\
\hline
\end{tabular}

${ }^{\text {a }}$ NS: indicates no statistical significance among mean values at $P \leq 0.1$.

included: total nitrogen (TN), nitrate-N $\left(\mathrm{NO}_{3}-\mathrm{N}\right)$, ammonium-N $\left(\mathrm{NH}_{4}-\mathrm{N}\right)$, particulate organic matter carbon (POM-C), extractable sulfur (S), $\mathrm{NH}_{4} \mathrm{OAc}$ exchangeable $\mathrm{Ca}, \mathrm{Mg}$, and $\mathrm{Na}$ (Ex-Ca, Ex-Mg, and Ex-Na, respectively), cation exchange capacity (CEC), percent base saturation (BS) and percent $\mathrm{K}, \mathrm{Ca}, \mathrm{Mg}$, and $\mathrm{H}$ saturation.

Probability levels ( $\mathrm{Pr}>\mathrm{F}$ ) from an analysis of variance (ANOVA) for all 23 indicators at both sampling depths are presented in Appendix Tables A.1 and A.2 for the CC and C/S fields, respectively.
There were several statistically significant $(P \leq 0.1)$ tillage, texture group, and replicate main effects, but the only tillage by texture group interactions that were statistically significant were for $\mathrm{pH}$, Bray $\mathrm{P}$ and $\mathrm{MBC}$ at the $0-5-\mathrm{cm}$ depth and for extractable $\mathrm{S}$ at the 515-cm depth.

Mean CEC, total base saturation, and saturation percentages for $\mathrm{K}, \mathrm{Ca}, \mathrm{Mg}$, and $\mathrm{H}$ are presented in Tables 5 and 6 . Although there are currently no SMAF scoring curves for these indicators, they were

Table 6

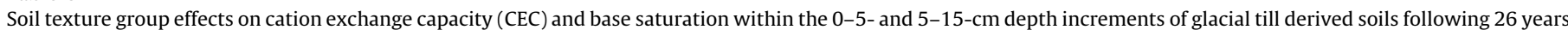
of continuous corn $(\mathrm{CC})$ or 31 years of a corn-soybean $(\mathrm{C} / \mathrm{S})$ rotation in central Iowa.

\begin{tabular}{|c|c|c|c|c|c|c|}
\hline \multirow[t]{2}{*}{ Texture group } & \multirow{2}{*}{$\begin{array}{l}\text { CEC } \\
\left(\mathrm{cmol} \mathrm{kg}^{-1}\right)\end{array}$} & \multicolumn{5}{|c|}{ Base saturation } \\
\hline & & $\begin{array}{l}\text { Total } \\
\left(\mathrm{g} \mathrm{kg}^{-1}\right)\end{array}$ & $\begin{array}{l}\mathrm{K} \\
\left(\mathrm{g} \mathrm{kg}^{-1}\right)\end{array}$ & $\begin{array}{l}\mathrm{Ca} \\
\left(\mathrm{g} \mathrm{kg}^{-1}\right)\end{array}$ & $\begin{array}{l}\mathrm{Mg} \\
\left(\mathrm{g} \mathrm{kg}^{-1}\right)\end{array}$ & $\begin{array}{l}\mathrm{H} \\
\left(\mathrm{g} \mathrm{kg}^{-1}\right)\end{array}$ \\
\hline & \multicolumn{6}{|c|}{ CC: $0-5 \mathrm{~cm}$ depth } \\
\hline Loam & 17 & 942 & 21 & 748 & 173 & 58 \\
\hline Clay loam & 20 & 895 & 19 & 701 & 175 & 105 \\
\hline \multirow[t]{2}{*}{$\operatorname{LSD}_{(0.1)}$} & 1 & 42 & $\mathrm{NS}^{\mathrm{a}}$ & 37 & NS & 42 \\
\hline & \multicolumn{6}{|c|}{ CC: $5-15 \mathrm{~cm}$ depth } \\
\hline Loam & 16 & 946 & 14 & 755 & 177 & 54 \\
\hline Clay loam & 19 & 896 & 12 & 707 & 178 & 104 \\
\hline \multirow[t]{2}{*}{$\operatorname{LSD}_{(0.1)}$} & 1 & NS & 1 & NS & NS & NS \\
\hline & \multicolumn{6}{|c|}{ C/S: $0-5 \mathrm{~cm}$ depth } \\
\hline Loam & 16 & 884 & 17 & 692 & 174 & 116 \\
\hline Clay loam & 20 & 855 & 17 & 652 & 186 & 145 \\
\hline \multirow[t]{2}{*}{$\operatorname{LSD}_{(0.1)}$} & 1 & NS & NS & 32 & NS & NS \\
\hline & \multicolumn{6}{|c|}{ C/S: $5-15 \mathrm{~cm}$ depth } \\
\hline Loam & 16 & 829 & 16 & 652 & 161 & 171 \\
\hline Clay loam & 19 & 870 & 13 & 680 & 177 & 130 \\
\hline $\operatorname{LSD}_{(0.1)}$ & 1 & NS & 1 & NS & NS & NS \\
\hline
\end{tabular}

a NS: indicates no statistical significance among mean values at $P \leq 0.1$. 
Table 7

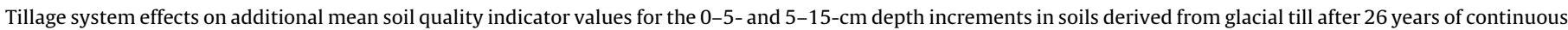
corn (CC) or 31 years of a corn-soybean $(C / S)$ rotation in central Iowa.

\begin{tabular}{|c|c|c|c|c|c|c|c|c|}
\hline Tillage system & $\mathrm{Ex}-\mathrm{Ca}^{\mathrm{a}}$ & $\begin{array}{l}\mathrm{Ex}-\mathrm{Mg} \\
\left(\mathrm{mg} \mathrm{kg}^{-1}\right)\end{array}$ & $\begin{array}{l}\mathrm{Ex}-\mathrm{Na} \\
\left(\mathrm{mg} \mathrm{kg}^{-1}\right)\end{array}$ & $\begin{array}{l}\mathrm{S} \\
\left(\mathrm{mg} \mathrm{kg}^{-1}\right)\end{array}$ & $\begin{array}{l}\mathrm{TN} \\
\left(\mathrm{mg} \mathrm{kg}^{-1}\right)\end{array}$ & $\begin{array}{l}\mathrm{NO}_{3}-\mathrm{N} \\
\left(\mathrm{mg} \mathrm{kg}^{-1}\right)\end{array}$ & $\begin{array}{l}\mathrm{NH}_{4}-\mathrm{N} \\
\left(\mathrm{mg} \mathrm{kg}^{-1}\right)\end{array}$ & $\begin{array}{l}\text { POM-C } \\
\left(\mathrm{mgg}^{-1}\right)\end{array}$ \\
\hline & \multicolumn{8}{|c|}{ CC: $0-5 \mathrm{~cm}$ depth } \\
\hline Moldboard plow & 2546 & 401 & 34 & 1.8 & 1.7 & 8 & 0.9 & 2.7 \\
\hline Chisel plow & 2735 & 402 & 32 & 2.1 & 2.0 & 14 & 2.1 & 4.8 \\
\hline Spring disk & 3075 & 343 & 32 & 1.8 & 2.2 & 16 & 1.2 & 7.4 \\
\hline Ridge-tillage & 2519 & 398 & 30 & 2.1 & 2.3 & 19 & 3.9 & 8.1 \\
\hline No-tillage & 2546 & 412 & 31 & 2.9 & 2.5 & 17 & 4.0 & 7.5 \\
\hline \multirow[t]{2}{*}{$\operatorname{LSD}_{(0.1)}$} & 285 & 46 & 2 & 0.6 & 0.1 & 3 & $N S^{b}$ & 1.4 \\
\hline & \multicolumn{8}{|c|}{ CC: $5-15 \mathrm{~cm}$ depth } \\
\hline Moldboard plow & 2346 & 390 & 34 & 2.6 & 1.6 & 6 & 0.8 & 1.7 \\
\hline Chisel plow & 2655 & 372 & 33 & 1.8 & 1.9 & 8 & 1.0 & 2.5 \\
\hline Spring disk & 2707 & 340 & 32 & 1.7 & 1.9 & 8 & 1.3 & 3.5 \\
\hline Ridge-tillage & 2584 & 404 & 33 & 2.5 & 1.8 & 6 & 2.9 & 1.9 \\
\hline No-tillage & 2670 & 392 & 34 & 1.9 & 1.9 & 7 & 1.4 & 1.9 \\
\hline \multirow[t]{2}{*}{$\operatorname{LSD}_{(0.1)}$} & 267 & 43 & NS & 0.8 & 0.2 & 1 & 1.9 & 1.2 \\
\hline & \multicolumn{8}{|c|}{ C/S: $0-5 \mathrm{~cm}$ depth } \\
\hline Moldboard plow & 2485 & 396 & 37 & 2.7 & 1.7 & 7 & 1.3 & 2.9 \\
\hline Chisel plow & 2489 & 404 & 35 & 2.7 & 2.0 & 11 & 0.7 & 5.1 \\
\hline Spring disk & 2607 & 428 & 34 & 2.6 & 2.2 & 14 & 0.8 & 6.2 \\
\hline Ridge-tillage & 2509 & 393 & 37 & 2.2 & 2.2 & 13 & 1.8 & 6.8 \\
\hline No-tillage & 2449 & 427 & 34 & 2.5 & 2.3 & 12 & 1.3 & 6.7 \\
\hline \multirow[t]{2}{*}{$\operatorname{LSD}_{(0.1)}$} & NS & NS & 2 & NS & 0.2 & 2 & 0.7 & 1.1 \\
\hline & \multicolumn{8}{|c|}{ C/S: $5-15 \mathrm{~cm}$ depth } \\
\hline Moldboard plow & 2348 & 367 & 35 & 2.5 & 1.7 & 6 & 1.4 & 3.4 \\
\hline Chisel plow & 2394 & 350 & 36 & 2.4 & 1.8 & 7 & 0.8 & 3.7 \\
\hline Spring disk & 2421 & 399 & 36 & 2.1 & 1.9 & 8 & 0.4 & 2.7 \\
\hline Ridge-tillage & 2414 & 357 & 36 & 2.3 & 1.8 & 5 & 0.7 & 2.7 \\
\hline No-tillage & 2482 & 371 & 37 & 2.2 & 1.9 & 6 & 2.0 & 2.4 \\
\hline $\operatorname{LSD}_{(0.1)}$ & NS & 43 & NS & NS & NS & 1 & 1.6 & NS \\
\hline
\end{tabular}

${ }^{\text {a }}$ Ex-Ca: ammonium acetate exchangeable calcium; Ex-K: ammonium acetate exchangeable potassium; Ex-Na: ammoniuim acetate exchangeable sodium; S: hot water extractable sulfur; $\mathrm{TN}$ : total Kjeldahl nitrogen; $\mathrm{NO}_{3}-\mathrm{N}$ : nitrate nitrogen; $\mathrm{NH}_{4}-\mathrm{N}$ : ammonium nitrogen; POM-C: particulate organic matter carbon.

b NS: indicates no statistical significance among mean values at $P \leq 0.1$.

included in this long-term assessment of tillage and cropping systems because previous on-farm studies with similar soils (Karlen et al., 2002) had indicated K saturation was often less than the $20-50 \mathrm{~g} \mathrm{~kg}^{-1}$ suggested by those favoring use of the basic cation saturation ratio (BCSR) concept (Eckert, 1987) for developing fertilizer recommendations for Midwestern soils. Furthermore, other long-term Midwestern studies (e.g. Barak et al., 1997; Liu et al., 1997; Russell et al., 2006) have shown significant effects on
CEC and base saturation due to ammonia-based $\mathrm{N}$ fertilizer applications, which for 1976 through 2006 totaled 5022 and $2956 \mathrm{~kg} \mathrm{ha}^{-1}$ for the CC and C/S fields, respectively.

Among the five tillage systems (Table 5) CEC was significantly lower within the 5-15-cm depth increment in both the CC and C/S fields after long-term moldboard plowing and for the $0-5-\mathrm{cm}$ increment in the $\mathrm{C} / \mathrm{S}$ field. Also, except for the $0-5-\mathrm{cm}$ depth increment in the spring disk and no-till treatments, $\mathrm{K}$ saturation in

Table 8

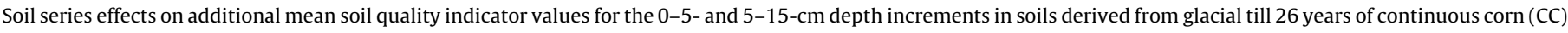
or 31 years of a corn-soybean $(C / S)$ rotation in central Iowa.

\begin{tabular}{|c|c|c|c|c|c|c|c|c|}
\hline Texture group & $\begin{array}{l}\mathrm{Ex}^{-\mathrm{Ca}^{\mathrm{a}}} \\
\left(\mathrm{mg} \mathrm{kg}^{-1}\right)\end{array}$ & $\begin{array}{l}\text { Ex-Mg } \\
\left(\mathrm{mg} \mathrm{kg}^{-1}\right)\end{array}$ & $\begin{array}{l}\text { Ex-Na } \\
\left(\mathrm{mg} \mathrm{kg}^{-1}\right)\end{array}$ & $\begin{array}{l}\mathrm{S} \\
\left(\mathrm{mg} \mathrm{kg}^{-1}\right)\end{array}$ & $\begin{array}{l}\text { TN } \\
\left(\mathrm{mg} \mathrm{kg}^{-1}\right)\end{array}$ & $\begin{array}{l}\mathrm{NO}_{3}-\mathrm{N} \\
\left(\mathrm{mg} \mathrm{kg}^{-1}\right)\end{array}$ & $\begin{array}{l}\mathrm{NH}_{4}-\mathrm{N} \\
\left(\mathrm{mg} \mathrm{kg}^{-1}\right)\end{array}$ & $\begin{array}{l}\text { POM-C } \\
\left(\mathrm{mgg}^{-1}\right)\end{array}$ \\
\hline & \multicolumn{8}{|c|}{ CC: $0-5 \mathrm{~cm}$ depth } \\
\hline Loam & 2608 & 358 & 32 & 1.9 & 2.0 & 12 & 1.3 & 5.8 \\
\hline Clay loam & 2744 & 413 & 32 & 2.3 & 2.2 & 16 & 3.1 & 6.6 \\
\hline \multirow[t]{2}{*}{$\operatorname{LSD}_{(0.1)}$} & $N S^{\mathrm{b}}$ & 30 & NS & 0.4 & 0.1 & 2 & NS & NS \\
\hline & \multicolumn{8}{|c|}{ CC: $5-15 \mathrm{~cm}$ depth } \\
\hline Loam & 2409 & 338 & 33 & 1.7 & 1.7 & 7 & 0.7 & 2.3 \\
\hline Clay loam & 2717 & 407 & 34 & 2.3 & 1.9 & 7 & 2.0 & 2.4 \\
\hline \multirow[t]{2}{*}{$\operatorname{LSD}_{(0.1)}$} & 172 & 27 & NS & 0.5 & 0.1 & NS & 1.2 & NS \\
\hline & \multicolumn{8}{|c|}{ C/S: $0-5 \mathrm{~cm}$ depth } \\
\hline Loam & 2263 & 338 & 34 & 2.4 & 1.8 & 10 & 0.7 & 5.0 \\
\hline Clay loam & 2654 & 452 & 36 & 2.6 & 2.2 & 12 & 1.5 & 5.9 \\
\hline \multirow[t]{2}{*}{$\operatorname{LSD}_{(0.1)}$} & 186 & 31 & 1 & NS & 0.1 & 2 & 0.5 & 0.8 \\
\hline & \multicolumn{8}{|c|}{ C/S: $5-15 \mathrm{~cm}$ depth } \\
\hline Loam & 2082 & 306 & 34 & 2.2 & 1.6 & 6.2 & 0.8 & 2.5 \\
\hline Clay loam & 2610 & 407 & 37 & 2.4 & 2.0 & 6.5 & 1.2 & 3.4 \\
\hline $\operatorname{LSD}_{(0.1)}$ & 156 & 28 & 1 & NS & 0.1 & 0.7 & NS & NS \\
\hline
\end{tabular}

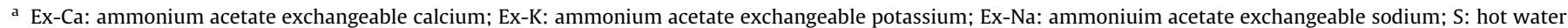
extractable sulfur; TN: total Kjeldahl nitrogen; $\mathrm{NO}_{3}-\mathrm{N}$ : nitrate nitrogen; $\mathrm{NH}_{4}-\mathrm{N}$ : ammonium nitrogen; POM-C: particulate organic matter carbon.

b NS: indicates no statistical significance among mean values at $P \leq 0.1$. 
the CC field was less than the suggested BCSR level of $20-50 \mathrm{~g} \mathrm{~kg}^{-1}$. Comparisons between soil texture groups (Table 6) show significantly lower CEC values for the loam sites than for clay loam sites for both sampling depths following either a long-term $\mathrm{CC}$ or $\mathrm{C} / \mathrm{S}$ rotation. Again, except for the loam 0-5-cm mean under $\mathrm{CC}, \mathrm{K}$ saturation was below $20 \mathrm{~g} \mathrm{~kg}^{-1}$. Low $\mathrm{K}$ saturation was especially evident within the $5-15-\mathrm{cm}$ depth increment, and was one reason that supplemental studies focused on soil-test $\mathrm{K}$ were initiated at this site (Kovar et al., 2009). Presumably the very high $\mathrm{Ca}$ and $\mathrm{Mg}$ levels (Tables 5 and 6), which are characteristic of glacial till derived soils within the land form known as the Des Moines lobe (Prior, 1991), further aggravated the low soil-test K levels and contributed to the plant K deficiencies noted by Kovar et al. (2009).

Tillage system and soil texture group differences for exchangeable $\mathrm{Ca}, \mathrm{Mg}$, and $\mathrm{Na}$, extractable $\mathrm{S}, \mathrm{TN}, \mathrm{NH}_{4}-\mathrm{N}, \mathrm{NO}_{3}-\mathrm{N}$, and POM-C are presented in Tables 7 and 8 . For several of these potential soil quality indicators, there were statistical differences among tillage systems and between texture groups, but the magnitude of those differences were small and are considered to be insignificant with regard to crop growth, development or yield. The most consistent difference among the five tillage systems was that TN in samples from both depth increments within moldboard plow plots were lower than for the other tillage systems. The POM-C levels were also lowest for the moldboard plow treatment within the $0-5-\mathrm{cm}$ increment after long-term CC or C/S rotations. Those differences are consistent with those discussed for total organic carbon (Table 1) and presumably reflect the lower soil organic matter levels in moldboard plow treatments. Unfortunately, baseline soil organic matter data were not collected when the study was initiated in 1975 , so it is not possible to discern if the lower TOC levels in the moldboard plow treatments are due to the long-term tillage or inherent differences. Between the two soil texture groups, exchangeable $\mathrm{Ca}$ and $\mathrm{Mg}$ were, as expected, significantly higher at clay loam sites than at the more sloping loam sites. Also, as expected, POM-C levels in the $0-5-\mathrm{cm}$ depth increment were generally twice as high as in the 5 to $15-\mathrm{cm}$ increment, again reflecting stratification and a rapid decline in soil carbon as sampling depth increases.

\section{Conclusions}

This study confirmed our hypothesis that long-term moldboard plowing and its associated secondary tillage operations would have the most negative impact on several soil quality/health indicators, especially those associated with TOC or one of the carbon fractions. In addition to TOC, CEC, WSA, MBC, and PMN all had lower values and/or SMAF scores in samples representing both texture groups where moldboard plowing had been used for either CC or C/S production.

This study also confirmed that low K saturation was very likely a chemical soil quality problem and supports conclusions by Karlen et al. (2013) that additional $\mathrm{K}$ fertility research is needed on glacial-till derived soils. Overall, based on our SMAF analysis, soils at this site were found to be functioning at between 75 and $85 \%$ of their potential. We conclude that developing less aggressive tillage systems or cropping systems that require less tillage could significantly improve soil quality/health even for some of the most productive soils in the world.

\section{Acknowledgements}

Numerous USDA-ARS and Iowa State University (ISU) research support personnel have contributed to the care and maintenance of the plots and data associated with this long-term assessment. Without their dedication, this type of long-term, tillage system evaluation would not have been feasible.

\section{Appendix A. Appendix}

See Tables A.1 and A.2

Table A.1

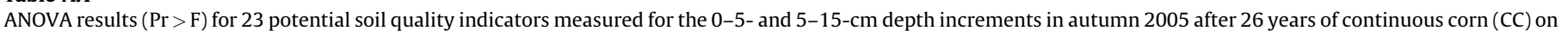
glacial till soils in central Iowa.

\begin{tabular}{|c|c|c|c|c|}
\hline Indicator & Tillage $(\mathrm{T})$ & Texture & Replicate & $\mathrm{T} \times$ Texture \\
\hline & \multicolumn{4}{|c|}{$0-5 \mathrm{~cm}$} \\
\hline Aggregate stability (WSA) & 0.0002 & 0.0001 & 0.0757 & 0.1710 \\
\hline Bulk density (BD) & $<0.0001$ & 0.0010 & 0.8286 & 0.1837 \\
\hline $\mathrm{pH}$ & 0.0090 & 0.1175 & 0.0313 & 0.0422 \\
\hline Electrical conductivity (EC) & 0.0144 & 0.2382 & 0.0263 & 0.5542 \\
\hline Bray P & $<0.0001$ & $<0.0001$ & $<0.0001$ & 0.0532 \\
\hline $\mathrm{NH}_{4} \mathrm{OAc}$ exchangeable $\mathrm{K}(\mathrm{Ex}-\mathrm{K})$ & 0.3580 & 0.0006 & 0.0016 & 0.8542 \\
\hline $\mathrm{NH}_{4} \mathrm{OAc}$ exchangeable $\mathrm{Ca}$ (Ex-Ca) & 0.8564 & 0.0100 & 0.0025 & 0.1559 \\
\hline $\mathrm{NH}_{4} \mathrm{OAc}$ exchangeable $\mathrm{Mg}(\mathrm{Ex}-\mathrm{Mg})$ & 0.8094 & $<0.0001$ & 0.0515 & 0.5040 \\
\hline $\mathrm{NH}_{4} \mathrm{OAc}$ exchangeable $\mathrm{Na}(\mathrm{Ex}-\mathrm{Na})$ & 0.0451 & 0.0680 & 0.1183 & 0.2184 \\
\hline CEC & 0.2693 & $<0.0001$ & 0.0108 & 0.1429 \\
\hline Extractable S & 0.3559 & 0.3072 & 0.6056 & 0.2311 \\
\hline Total N (TN) & 0.0002 & $<0.0001$ & 0.0085 & 0.2713 \\
\hline Nitrate $\mathrm{N}\left(\mathrm{NO}_{3}-\mathrm{N}\right)$ & 0.0002 & 0.0813 & 0.2365 & 0.3165 \\
\hline Ammonium $\mathrm{N}\left(\mathrm{NH}_{4}-\mathrm{N}\right)$ & 0.1395 & 0.1633 & 0.0372 & 0.3414 \\
\hline Total organic C (TOC) & 0.0012 & $<0.0001$ & 0.0096 & 0.4677 \\
\hline Microbial biomass C (MBC) & $<0.0001$ & $<0.0001$ & 0.0236 & 0.0483 \\
\hline Potential mineralizable $\mathrm{N}$ (PMN) & $<0.0001$ & 0.2350 & 0.3385 & 0.2106 \\
\hline Particulate organic matter (POM-C) & $<0.0001$ & 0.3082 & 0.7509 & 0.1955 \\
\hline Percent base saturation (BS) & 0.2510 & 0.0180 & 0.0158 & 0.2629 \\
\hline Percent K & 0.2720 & 0.3349 & 0.5425 & 0.1714 \\
\hline Percent Ca & 0.2696 & 0.0036 & 0.0183 & 0.1266 \\
\hline Percent Mg & 0.4359 & 0.4404 & 0.4146 & 0.1591 \\
\hline \multirow[t]{2}{*}{ Percent $\mathrm{H}$} & 0.2510 & 0.0180 & 0.0158 & 0.2629 \\
\hline & \multicolumn{4}{|c|}{$5-15 \mathrm{~cm}$} \\
\hline Aggregate stability (WSA) & 0.2561 & $<0.0001$ & 0.0033 & 0.2688 \\
\hline Bulk density (BD) & 0.5069 & 0.0567 & 0.2427 & 0.6334 \\
\hline $\mathrm{pH}$ & 0.0234 & 0.6067 & 0.0143 & 0.9060 \\
\hline
\end{tabular}


Table A.1 (Continued)

\begin{tabular}{|c|c|c|c|c|}
\hline Indicator & Tillage $(\mathrm{T})$ & Texture & Replicate & $\mathrm{T} \times$ Texture \\
\hline Electrical conductivity (EC) & 0.7664 & 0.9545 & 0.0597 & 0.7321 \\
\hline Bray P & 0.3187 & 0.0003 & 0.0008 & 0.4336 \\
\hline $\mathrm{NH}_{4} \mathrm{OAc}$ exchangeable $\mathrm{K}(\mathrm{Ex}-\mathrm{K})$ & 0.5906 & 0.6637 & $<0.0001$ & 0.7962 \\
\hline $\mathrm{NH}_{4} \mathrm{OAc}$ exchangeable $\mathrm{Ca}(\mathrm{Ex}-\mathrm{Ca})$ & 0.8402 & $<0.0001$ & 0.0009 & 0.7535 \\
\hline $\mathrm{NH}_{4} \mathrm{OAc}$ exchangeable $\mathrm{Mg}$ (Ex-Mg) & 0.9725 & $<0.0001$ & 0.0011 & 0.3930 \\
\hline $\mathrm{NH}_{4} \mathrm{OAc}$ exchangeable $\mathrm{Na}(\mathrm{Ex}-\mathrm{Na})$ & 0.8775 & 0.0025 & 0.0805 & 0.4909 \\
\hline CEC & 0.2460 & 0.0004 & 0.0158 & 0.8977 \\
\hline Extractable S & 0.2897 & 0.2290 & 0.8109 & 0.0490 \\
\hline Total N (TN) & 0.9310 & $<0.0001$ & 0.0043 & 0.8985 \\
\hline Nitrate $\mathrm{N}\left(\mathrm{NO}_{3}-\mathrm{N}\right)$ & 0.0012 & 0.0674 & 0.5300 & 0.7375 \\
\hline Ammonium $\mathrm{N}\left(\mathrm{NH}_{4}-\mathrm{N}\right)$ & 0.7070 & 0.2621 & 0.0275 & 0.2962 \\
\hline Total organic C (TOC) & 0.6478 & $<0.0001$ & 0.0169 & 0.8472 \\
\hline Microbial biomass C (MBC) & 0.0418 & $<0.0001$ & 0.0893 & 0.2043 \\
\hline Potential mineralizable N (PMN) & 0.5407 & 0.4405 & 0.2092 & 0.1821 \\
\hline Particulate organic matter (POM-C) & 0.1700 & 0.0646 & 0.0227 & 0.1362 \\
\hline Percent base saturation (BS) & 0.0818 & 0.6182 & 0.0263 & 0.8603 \\
\hline Percent K & 0.5873 & 0.0029 & 0.2299 & 0.4394 \\
\hline Percent Ca & 0.1009 & 0.6789 & 0.0374 & 0.9192 \\
\hline Percent Mg & 0.1034 & 0.3471 & 0.0842 & 0.5814 \\
\hline Percent $\mathrm{H}$ & 0.0818 & 0.6182 & 0.0263 & 0.8603 \\
\hline
\end{tabular}

Table A.2

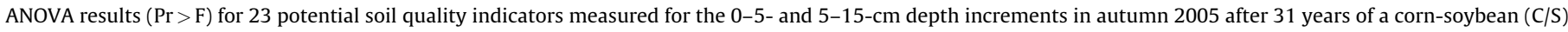
rotation on glacial till soils in central Iowa.

\begin{tabular}{|c|c|c|c|c|}
\hline Indicator & Tillage $(\mathrm{T})$ & Texture & Replicate & $\mathrm{T} \times$ Texture \\
\hline & \multicolumn{4}{|c|}{$0-5 \mathrm{~cm}$} \\
\hline Aggregate stability (WSA) & 0.0002 & 0.0001 & 0.0757 & 0.1710 \\
\hline Bulk density (BD) & $<0.0001$ & 0.0010 & 0.8286 & 0.1837 \\
\hline $\mathrm{pH}$ & 0.0090 & 0.1175 & 0.0313 & 0.0422 \\
\hline Electrical conductivity (EC) & 0.0144 & 0.2382 & 0.0263 & 0.5542 \\
\hline Bray $\mathrm{P}$ & $<0.0001$ & $<0.0001$ & $<0.0001$ & 0.0532 \\
\hline $\mathrm{NH}_{4} \mathrm{OAc}$ exchangeable $\mathrm{K}$ (Ex-K) & 0.3580 & 0.0006 & 0.0016 & 0.8542 \\
\hline $\mathrm{NH}_{4} \mathrm{OAc}$ exchangeable $\mathrm{Ca}(\mathrm{Ex}-\mathrm{Ca})$ & 0.8564 & 0.0100 & 0.0025 & 0.1559 \\
\hline $\mathrm{NH}_{4} \mathrm{OAc}$ exchangeable $\mathrm{Mg}(\mathrm{Ex}-\mathrm{Mg})$ & 0.8094 & $<0.0001$ & 0.0515 & 0.5040 \\
\hline $\mathrm{NH}_{4} \mathrm{OAc}$ exchangeable $\mathrm{Na}(\mathrm{Ex}-\mathrm{Na})$ & 0.0451 & 0.0680 & 0.1183 & 0.2184 \\
\hline CEC & 0.2693 & $<0.0001$ & 0.0108 & 0.1429 \\
\hline Extractable S & 0.3559 & 0.3072 & 0.6056 & 0.2311 \\
\hline Total N (TN) & 0.0002 & $<0.0001$ & 0.0085 & 0.2713 \\
\hline Nitrate $\mathrm{N}\left(\mathrm{NO}_{3}-\mathrm{N}\right)$ & 0.0002 & 0.0813 & 0.2365 & 0.3165 \\
\hline Ammonium $\mathrm{N}\left(\mathrm{NH}_{4}-\mathrm{N}\right)$ & 0.1395 & 0.1633 & 0.0372 & 0.3414 \\
\hline Total organic C (TOC) & 0.0012 & $<0.0001$ & 0.0096 & 0.4677 \\
\hline Microbial biomass C (MBC) & $<0.0001$ & $<0.0001$ & 0.0236 & 0.0483 \\
\hline Potential mineralizable N (PMN) & $<0.0001$ & 0.2350 & 0.3385 & 0.2106 \\
\hline Particulate organic matter (POM-C) & $<0.0001$ & 0.3082 & 0.7509 & 0.1955 \\
\hline Percent base saturation (BS) & 0.2434 & 0.1759 & 0.0057 & 0.2629 \\
\hline Percent K & 0.2720 & 0.3349 & 0.5425 & 0.1714 \\
\hline Percent Ca & 0.2696 & 0.0036 & 0.0183 & 0.1266 \\
\hline Percent Mg & 0.4359 & 0.4404 & 0.4146 & 0.1591 \\
\hline \multirow[t]{2}{*}{ Percent $\mathrm{H}$} & 0.2434 & 0.1759 & 0.0057 & 0.2629 \\
\hline & \multicolumn{4}{|c|}{$5-15 \mathrm{~cm}$} \\
\hline Aggregate stability (WSA) & 0.2561 & $<0.0001$ & 0.0033 & 0.2688 \\
\hline Bulk density (BD) & 0.5069 & 0.0567 & 0.2427 & 0.6334 \\
\hline $\mathrm{pH}$ & 0.0234 & 0.6067 & 0.0143 & 0.9060 \\
\hline Electrical conductivity (EC) & 0.7664 & 0.9545 & 0.0597 & 0.7321 \\
\hline Bray P & 0.3187 & 0.0003 & 0.0008 & 0.4336 \\
\hline $\mathrm{NH}_{4} \mathrm{OAc}$ exchangeable $\mathrm{K}(\mathrm{Ex}-\mathrm{K})$ & 0.5906 & 0.6637 & $<0.0001$ & 0.7962 \\
\hline $\mathrm{NH}_{4} \mathrm{OAc}$ exchangeable $\mathrm{Ca}(\mathrm{Ex}-\mathrm{Ca})$ & 0.8402 & $<0.0001$ & 0.0009 & 0.7535 \\
\hline $\mathrm{NH}_{4} \mathrm{OAc}$ exchangeable $\mathrm{Mg}$ (Ex-Mg) & 0.9725 & $<0.0001$ & 0.0011 & 0.3930 \\
\hline $\mathrm{NH}_{4} \mathrm{OAc}$ exchangeable $\mathrm{Na}(\mathrm{Ex}-\mathrm{Na})$ & 0.8775 & 0.0025 & 0.0805 & 0.4909 \\
\hline CEC & 0.2460 & 0.0004 & 0.0158 & 0.8977 \\
\hline Extractable S & 0.2897 & 0.2290 & 0.8109 & 0.0490 \\
\hline Total N (TN) & 0.9310 & $<0.0001$ & 0.0043 & 0.8985 \\
\hline Nitrate $\mathrm{N}\left(\mathrm{NO}_{3}-\mathrm{N}\right)$ & 0.0012 & 0.0674 & 0.5300 & 0.7375 \\
\hline Ammonium $\mathrm{N}\left(\mathrm{NH}_{4}-\mathrm{N}\right)$ & 0.7070 & 0.2621 & 0.0275 & 0.2962 \\
\hline Total organic C (TOC) & 0.6478 & $<0.0001$ & 0.0169 & 0.8472 \\
\hline Microbial biomass C (MBC) & 0.0418 & $<0.0001$ & 0.0893 & 0.2043 \\
\hline Potential mineralizable N (PMN) & 0.5407 & 0.4405 & 0.2092 & 0.1821 \\
\hline Particulate organic matter (POM-C) & 0.1700 & 0.0646 & 0.0227 & 0.1362 \\
\hline Percent base saturation (BS) & 0.0818 & 0.6182 & 0.0263 & 0.8603 \\
\hline Percent K & 0.5873 & 0.0029 & 0.2299 & 0.4394 \\
\hline Percent Ca & 0.1009 & 0.6789 & 0.0374 & 0.9192 \\
\hline Percent Mg & 0.1034 & 0.3471 & 0.0842 & 0.5814 \\
\hline Percent $\mathrm{H}$ & 0.0818 & 0.6182 & 0.0263 & 0.8603 \\
\hline
\end{tabular}




\section{References}

Alberts, E.E., Wendt, R.C., Burwell, R.E., 1985. Corn and soybean cropping effects on soil losses and C factors. Soil Science Society of America Journal 49, 721-728.

Andrews, S.S., Karlen, D.L., Cambardella, C.A., 2004. The soil management assessment framework: a quantitative soil quality evaluation method. Soil Science Society of America Journal 68, 1945-1962.

Arshad, M.A., Lowery, B., Grossman, B., 1996. Physical tests for monitoring soil quality In: Doran, J.W., Jones, A.J. (Eds.), Methods for Assessing Soil Quality. Special Publication Number 49. Soil Science Society of America, Inc., Madison, WI, pp. $123-141$

Barak, P., Jobe, B.O., Krueger, A.R., Peterson, L.A., Laird, D.A., 1997. Effects of longterm soil acidification due to nitrogen fertilizer inputs in Wisconsin. Plant and Soil 197, 61-69.

Blake, G.R., Hartge, K.H., 1986. Bulk density. In: Klute, A. (Ed.), Methods of Soil Analysis. Part 1. 2nd ed. Agronomy Monograph Number 9. American Society of Agronomy and Soil Science Society of America, Madison, WI, pp. 363-375.

Bray, R.H., Kurtz, L.T., 1945. Determination of total, organic and available form of phosphorus in soil. Soil Science Society of America Proceedings 59, 39-45.

Cambardella, C.A., Elliott, E.T., 1992. Particulate soil organic-matter changes across a grassland cultivation sequence. Soil Science Society of America Journal 56, 777-783.

Chase, S., 1936. Rich Land, Poor Land: A Study of Waste in the Natural Resources of America. Whittlesey House/McGraw-Hill, New York, pp. 361.

Derpsch, R., 1998. Historical review of no-tillage cultivation of crops. In: Proceedings of 1st Japan International Research Center for Agricultural Sciences (JIRCAS) Seminar on Soybean Research. No-Tillage Cultivation and Future Research Needs, March 5-6, 1998, Iguassu Falls, Brazil. JIRCAS Working Report No. 13, pp. $1-19$.

Drinkwater, L.E., Cambardella, C.A., Reeder, J.D., Rice, C.W., 1996. Potentially mineralizable nitrogen as an indicator of biologically active soil nitrogen. In: Doran, J.W., Jones, A.J. (Eds.), Methods for Assessing Soil Quality. Soil Science Society of America (SSSA) Special Publication Number 49. SSSA, Inc., Madison, WI, pp. 217-229.

Eckert, D.J., 1987. Soil test interpretations: basic cation saturation ratios and sufficiency levels. In: Brown, J.R. (Ed.), Soil Testing: Sampling, Correlation, Calibration, and interpretation. Soil Science Society of America (SSSA) Special Publication No. 21. SSSA, Madison, WI, pp. 53-64.

Erbach, D.C., 1982. Tillage for continuous corn and corn-soybean rotation. Transactions of the American Society of Agricultural Engineering (ASAE) 25, 906-918.

Fernandez-Ugale, O., Virto, I., Bescansa, P., Imaz, M.J., Enrique, A., Karlen, D.L., 2009. No-tillage improvement of soil physical quality in calcareous, degradationprone, semiarid soils. Soil \& Tillage Research 106, 29-35.

Imaz, M.J., Virto, I., Bescansa, P., Enrique, A., Fernandez-Ugalde, O., Karlen, D.L., 2010. Soil quality indicator response to tillage and residue management on semi-arid Mediterranean cropland. Soil \& Tillage Research 107, 17-25.

Jokela, W.E., Grabber, J.H., Karlen, D.L., Balser, T.C., Palmquist, D.E., 2009. Cover crop and liquid manure effects on soil quality indicators in a corn silage system. Agronomy Journal 101, 727-737.

Karlen, D.L., Mausbach, M.J., Doran, J.W., Cline, R.G., Harris, R.F., Schuman, G.E., 1997. Soil quality: a concept, definition, and framework for evaluation. Soil Science Society of America Journal 61, 4-10.

Karlen, D.L., Kohler, K.A., Laird, D.A., Thompson, R.L., Buhler, D.D., 2002. Soil-test dynamics throughout a five-year 'Thompson Farm' rotation in Iowa. American Journal of Alternative Agriculture 17, 9-17.

Karlen, D.L., Hurley, E.G., Andrews, S.S., Cambardella, C.A., Meek, D.W., Duffy, M.D., Mallarino, A.P., 2006. Crop rotation effects on soil quality at three northern corn/soybean belt locations. Agronomy Journal 98, 484-495.

Karlen, D.L., Andrews, S.S., Wienhold, B.J., Zobeck, T.M., 2008. Soil quality assessment: past, present and future. Journal of Integrated Biosciences 6 (1) 3-14.

Karlen, D.L., Dinnes, D.L., Singer, J.W., 2010. Midwest soil and water conservation: past, present and future. In: Zobeck, T.M., Schillinger, W.F. (Eds.), Soil and Water Conservation Advances in the US: Past Efforts - Future Outlook. Soil Science Society of America. Inc., Madison, WI, pp. 131-162.

Karlen, D.L., Varvel, G.E., Johnson, J.M.F., Baker, J.M., Osborne, S.L., Novak, J.M., Adler, P.R., Roth, G.W., Birrell, S.J., 2011. Monitoring soil quality to assess the sustainability of harvesting corn stover. Agronomy Journal 103, 288-295.

Karlen, D.L., Kovar, J.L., Cambardella, C.A., Colvin, T.S., 2013. Thirty-year tillage effects on crop yield and soil fertility indicators. Soil \& Tillage Research 130, 28-41.
Keeney, D.R., Bremner, J.M., 1967. Determination of isotope-ratio analysis of different forms of nitrogen in soils: VI. Mineralizable nitrogen. Soil Science Society of America Proceedings 31, 34-39.

Kovar, J.L., Bortolon, L., Karlen, D.L., 2009. Distribution of phosphorus and potassium following surface banding of fertilizer in conservation tillage systems. In: Proceedings of XVI International Plant Nutrition Colloquium. August 26-30, 2009. Sacramento, CA. 2009 Available at: http://repositories.cdlib.org/ipnc/xvi/1345.

Liebig, M., Carpenter-Boggs, L., Johnson, J.M.F., Wright, S., Barbour, N., 2006. Cropping system effects on soil biological characteristics in the Great Plains. Renewable Agricultural and Food Systems 21, 36-48.

Liu, Y.J., Laird, D.A., Barak, P., 1997. Release and fixation of ammonium and potassium under long-term fertility management. Soil Science Society of America Journal 61, 310-314.

Phillips, E.R., Phillips, S.H. (Eds.), 1984. No-Tillage Agriculture, Principles and Practices. Van Nostrand Reinhold Co., New York, p. 306.

Prior, J., 1991. Landforms of Iowa. University of Iowa Press, Iowa City, IA, pp. 168.

Russell, A.E., Laird, D.A., Mallarino, A.P., 2006. Nitrogen fertilization and cropping system impacts on soil quality in Midwestern Mollisols. Soil Science Society of America Journal 70, 249-255.

Sawyer, J.E., Mallarino, A.P., Killorn, R., Barnhart, S.K., 2011. A General Guide for Crop Nutrient and Limestone Recommendations in Iowa. PM-1688. Iowa State University Extension, Ames, IA Available online at: http://222.extension.iastate.edu/Publications?PM1688.pdf.

SAS Institute, 1990. SAA/STAT User's Guide. Version 6, 4th ed. SAS Inst., Cary, NC.

Soil Survey Staff, 2010. Natural Resources Conservation Service (NRCS). United States Department of Agriculture (USDA). Official Soil Series Descriptions Online at: http://soils.usda.gov/technical/classification/osd/index.html.

Sparling, G.P., West, A.W., 1988. A direct extraction method to estimate soil microbial C: calibration in situ using microbial respiration and $14 \mathrm{C}$ labeled cells. Soil Biology and Biochemistry 20, 337-677.

Stott, D.E., Andrews, S.S., Liebig, M.A., Wienhold, B.J., Karlen, D.L., 2010. Evaluation of $\beta$-glucosidase activity as a soil quality indicator for the soil management assessment framework (SMAF). Soil Science Society of America Journal 74, 107-119.

Stott, D.E., Cambardella, C.A., Wolf, R., Tomer, M.D., Karlen, D.L., 2011. A Soil quality assessment within the Iowa River south fork watershed. Soil Science Society of America Journal 75, 2271-2282.

USDA-Soil Conservation Service (SCS), 1981. Soil Survey of Boone County, Iowa. USDA-Natural Resources Conservation Service (NRCS), Washington, DC pp. 200.

Vance, E.C., Brookes, P.C., Jenkinson, D.S., 1987. An extraction method for measuring microbial biomass C. Soil Biology and Biochemistry 19, 703-707.

Varvel, G.E., Wilhelm, W.W., 2011. No-tillage increases soil profile carbon and nitrogen under long-term rainfed cropping systems. Soil \& Tillage Research 114, 28-36.

Wagner, S.W., Hanson, J.D., Olness, A. Voorhees, W.B., 1998. A volumetric inorganic carbon analysis system. Soil Science Society of America Journal 62, 690-693.

Warncke, D., Brown, J.R., 1998. Potassium and other basic cations. In: Brown, J.R. (Ed.), Recommended Chemical Soil Test Procedures for the North Central Region (NCR), NCR Publication Number 221 (Revised). Missouri Agricultural Experimental Station, Columbia, MO, pp. 31-34.

Watson, M.E., Brown, J.R., 1998. pH and lime requirement. In: Brown, J.R. (Ed.), Recommended Chemical Soil Test Procedures for the North Central Region (NCR), NCR Publication Number 221 (Revised). Missouri Agricultural Experimental Station, Columbia, MO, pp. 13-16.

Whitney, D.A., 1998. Soil salinity. In: Brown, J.R. (Ed.), Recommended Chemical Soil Test Procedures for the North Central Region (NCR), NCR Publication Number 221 (Revised). Missouri Agricultural Experimental Station, Columbia, MO, pp. 59-60.

Wienhold, B.J., Pikul Jr., J.L., Liebig, M.A., Mikha, M.M., Varvel, G.E., Doran, J.W., Andrews, S.S., 2006. Cropping system effects on soil quality in the Great Plains: synthesis from a regional project. Renewable Agricultural and Food Systems 21, 49-59.

Wienhold, B.J., Karlen, D.L., Andrews, S.S., Stott, D.E., 2009. Protocol for indicator scoring in the soil management assessment framework (SMAF). Renewable Agricultural and Food Systems 24, 260-266.

Yoder, R.E., 1936. A direct method of aggregate analysis of soils and a study of the physical nature of erosion losses. Journal of the American Society of Agronomy 28, 337-351.

Zobeck, T.M., Halvorson, A.D., Wienhold, B.J., Acosta Martinez, V., Karlen, D.L., 2008. Comparison of two soil quality indexes to evaluate cropping systems in northern Colorado. Journal of Soil and Water Conservation 63, 329-338. 